\title{
Adherence to fecal immunochemical test screening among adults at average risk for colorectal cancer
}

\author{
Deborah A. Fisher ${ }^{1} \cdot$ Nicole Princic $^{2} \cdot$ Lesley-Ann Miller-Wilson $^{3} \cdot$ Kathleen Wilson $^{2} \cdot$ Kathryn DeYoung $^{2}$. \\ A. Burak Ozbay ${ }^{3} \cdot$ Paul Limburg ${ }^{4}$
}

Accepted: 25 October 2021 / Published online: 2 November 2021

(c) The Author(s) 2021

\begin{abstract}
Purpose This study examined adherence to screening for fecal immunochemical test (FIT).

Methods Adults ( $\geq 50-75)$ with a FIT between 1/1/2014 and 6/30/2019 in MarketScan administrative claims were selected (index $=$ earliest FIT). Patients were followed for 10 years pre- and 3 years post-index. Patients at increased risk for CRC or with prior screening were excluded. Year over year adherence was measured post-index.

Results Of 10,253 patients, the proportion adherent to repeat testing at year 2 was $23.4 \%$ and $10.6 \%$ at year 3. Of $76.6 \%$ not adherent in year $2,5.4 \%$ were adherent in year 3 .

Conclusion Results suggest adherence to FIT tests is poor, minimizing potential benefits. Future studies are needed to consider alternative test options and whether more choice will improve long-term adherence.
\end{abstract}

Keywords Colorectal neoplasms $\cdot$ Mass screening $\cdot$ Guideline adherence

\section{Objective}

Guideline organizations such as the United States Preventive Services Task Force recommend that colorectal cancer (CRC) screening for average-risk, asymptomatic adults begin at age 50 and continue until age 75 [1]. Although colonoscopy remains the most frequently used screening test for CRC, the fecal immunochemical test (FIT) is a common non-invasive CRC screening option; however, the clinical effectiveness of this method is dependent on long-term adherence to annual testing [2, 3]. The present retrospective, claims-based study examined adherence to FIT over three consecutive cycles of annual screening among a population of screen eligible adults.

Nicole Princic

nprincic@us.ibm.com

1 Division of Gastroenterology, Duke University School of Medicine, 3100 Tower Blvd, Durham, NC 27707, USA

2 IBM Watson Health, 75 Binney St, Cambridge, MA 02142, USA

3 Exact Sciences Corporation, 441 Charmany Dr, Madison, WI 53719, USA

4 Division of Gastroenterology and Hepatology, Mayo Clinic, 200 1st St SW, Rochester, MN 55905, USA

\section{Methods}

Average-risk adults, ages 50-75 years, and who had a procedure code for FIT testing between January 1, 2014 and June 30, 2019 were identified in the IBM MarketScan Commercial and Medicare Supplemental Databases. The index date was the date of the first claim for FIT during the study eligibility period. Patients were required to have 10 years of continuous enrollment prior to the index date to access average risk status and to ensure patients were due for screening. To identify average-risk participants, we excluded participants with a history of benign or malignant colorectal neoplasms, inflammatory bowel disease, a known family history of gastrointestinal cancer, or evidence of blood in the stool in the three months preceding the index date. To ensure patients were due for screening, participants were required to have no evidence or FIT or fecal occult blood testing (FOBT) in the year prior to index date, no evidence of multi-target stool DNA test in 3 years prior to index date, no evidence of other screening (i.e., flexible sigmoidoscopy, CT colonography or double contrast barium enema) in 5 years prior to index date, and no evidence of colonoscopy in 10 years prior to index date. Although, this study was designed to assess non-invasive CRC screening modalities, however, due to sample size limitations, the 
final analysis was restricted to participants screened with FIT. In addition, participants with evidence of any non-FIT CRC screening modality during the 3-year data collection period used to measure adherence were excluded.

Adherence to FIT was examined over three time periods: index (T0), first follow-up screening window (T1), and second follow-up screening window (T2). FIT adherence in T1 was defined as completion of a FIT at months 12-15, allowing for a 3-month grace period for delayed screening 4. Adherence in T2 was defined as completion of a FIT at 12-15 months after the $\mathrm{T} 1$ screening (among those who completed T1 screening) or at months 16-36 among those who did not complete T1 screening. Adherence patterns were defined as consistently adherent (FIT at both $\mathrm{T} 1$ and $\mathrm{T} 2$ ), consistently nonadherent (no FIT at either T1 or T2), and partially adherent (FIT at only T1 or T2). The median months between tests were also reported.

Because this study used only de-identified medical records and did not involve the collection, use, or transmittal of individually identifiable data, it is not considered protected health information under the HIPAA Privacy Rule and is exempt from Institutional Review Board approval.

\section{Results}

In total, 10,253 participants met the defined study eligibility criteria. The mean participant age was 56.0 years, with sex distributions of $32.8 \%$ men. The large majority of participants were commercially insured: $94.7 \%$. Mean (SD) DeyoCharlson comorbidity score was $0.4(0.9)$.
For participants initiating FIT, 23.4\% were adherent in T1, while $76.6 \%$ were non-adherent in T1 (Fig. 1). Over the full follow-up, $10.6 \%$ of participants were consistently adherent, $72.4 \%$ were consistently nonadherent, and $17.0 \%$ were partially adherent. The median time between tests was 12.7 months.

\section{Discussion}

FIT screening combined with organized patient outreach has been shown to increase overall adherence to CRC screening and contribute to early detection and prevention of CRC [5]. However, reported longitudinal adherence rates vary. Repeat FIT adherence rates of $75.3 \%$ to $86.1 \%$ have been achieved in an organized screening program; [3] whereas, rates of $15.8 \%$ to $28.8 \%$ were reported from a safety-net health system [4]. In this study analyzing data from diverse health plans, $23.4 \%$ of participants had a repeat FIT in the first follow-up window, and only $10.6 \%$ of participants completed a repeat test during both follow-up windows.

This study is subject to the limitations common to all retrospective administrative claims studies, such as lack of independent confirmation that a test was completed. This study excluded those who switched screening modalities during the 3-year data collection period to ensure a measure of adherence the FIT test, however, it does not take into account that patients may be screened (and be adherent) using other test types after an initial FIT test.

Results from this analysis of robust claims data across many health plans suggest that longitudinal adherence to annual FIT is suboptimal for many insured individuals who

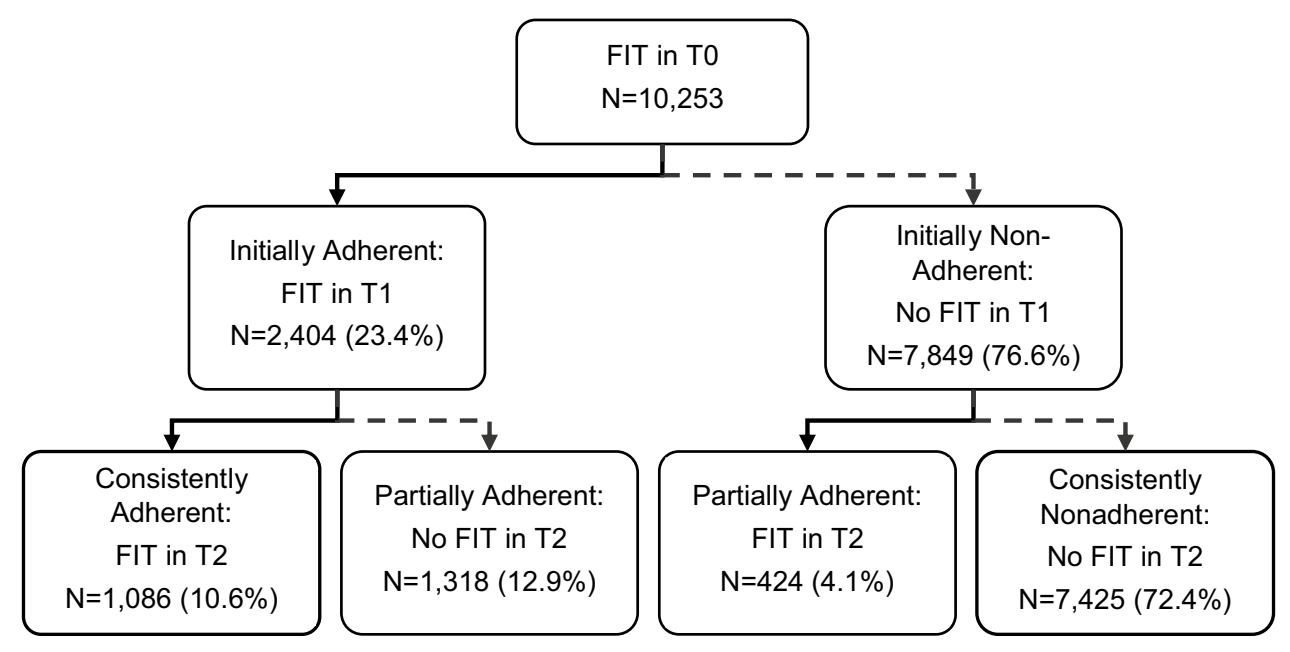

Fig. 1 Adherence to screening by fecal immunochemical testing (FIT). Adherence to FIT was defined as consistently adherent (FIT in both $\mathrm{T} 1$ and $\mathrm{T} 2$ ), consistently nonadherent (no FIT in either T1 or $\mathrm{T} 2$ ), and partially adherent (FIT in only T1 or T2). In total, $23.4 \%$ of participants had a repeat FIT in T1, and only $10.6 \%$ of participants completed a repeat FIT in T1 and T2. T0: Index FIT screening. T1: First follow-up screening window of months 12-15 after index FIT screening. T2: Second follow-up screening window of months 12-15 after FIT in T1 or months 16-36 after T0 (if no FIT in T1) 
initially pursue this screening strategy. Data reported from healthcare settings with organized screening programs suggest that considerable infrastructure and outreach is needed to achieve long term adherence within a FIT screening program [6]. Despite this, many modeling studies may assume an impractical, $100 \%$ adherence to FIT and other guidelineendorsed screening strategies, which does not reflect realworld, population-level experience. The data from this study can be used to develop models to more accurately estimate the potential benefits achievable with FIT-based screening strategies.

In conclusions, real-world adherence to FIT is suboptimal and this low adherence should be considered when developing models of CRC screening benefits. Future studies are needed to consider alternative CRC screening test options and whether more choice/flexibility will improve long-term adherence.

Acknowledgements Medical writing services were provided by Jessamine Winer-Jones, $\mathrm{PhD}$.

Author contribution All authors were involved in study design, analysis, and interpretation of the data, drafting and revising the paper, and approving the final version of the manuscript. All authors agree to be accountable for all aspects of the work.

Funding This study was funded by Exact Sciences Corporation.

\section{Declarations}

Conflict of interest Deborah A. Fisher was a consultant for Exact Sciences and Guardant Health. Nicole Princic and Kathleen Wilson are employees of IBM Watson Health. Lesley-Ann Miller-Wilson and A. Burak Ozbay are employees of Exact Sciences Corporation. Kathryn DeYoung was an employee of IBM Watson Health at the time the study was conducted. Paul Limburg serves as Chief Medical Officer for Screening at Exact Sciences through a contracted services agreement with Mayo Clinic. Dr. Limburg and Mayo Clinic have contractual rights to receive royalties through this agreement.
Open Access This article is licensed under a Creative Commons Attribution 4.0 International License, which permits use, sharing, adaptation, distribution and reproduction in any medium or format, as long as you give appropriate credit to the original author(s) and the source, provide a link to the Creative Commons licence, and indicate if changes were made. The images or other third party material in this article are included in the article's Creative Commons licence, unless indicated otherwise in a credit line to the material. If material is not included in the article's Creative Commons licence and your intended use is not permitted by statutory regulation or exceeds the permitted use, you will need to obtain permission directly from the copyright holder. To view a copy of this licence, visit http://creativecommons.org/licenses/by/4.0/.

\section{References}

1. U. S. Preventive Services Task Force (2016) Screening for colorectal cancer: US Preventive Services Task Force recommendation statement. JAMA 315(23):2564-75

2. Crotta S, Segnan N, Paganin S, Dagnes B, Rosset R, Senore C (2012) High rate of advanced adenoma detection in 4 rounds of colorectal cancer screening with the fecal immunochemical test. Clin Gastroenterol Hepatol 10(6):633-638

3. Jensen CD, Corley DA, Quinn VP et al (2016) Fecal immunochemical test program performance over 4 rounds of annual screening: a retrospective cohort study. Ann Intern Med 164(7):456-463

4. Murphy CC, Halm EA, Skinner CS, Balasubramanian BA, Singal AG (2020) Challenges and approaches to measuring repeat fecal immunochemical test for colorectal cancer screening. Cancer Epidemiol Biomarkers Prev 29(8):1557-1563

5. Levin TR, Corley DA, Jensen CD et al (2018) Effects of organized colorectal cancer screening on cancer incidence and mortality in a large community-based population. Gastroenterology 155(5):1383-91.e5

6. Selby K, Jensen CD, Levin TR et al (2020) Program components and results from an organized colorectal cancer screening program using annual fecal immunochemical testing. Clin Gastroenterol Hepatol

Publisher's Note Springer Nature remains neutral with regard to jurisdictional claims in published maps and institutional affiliations. 